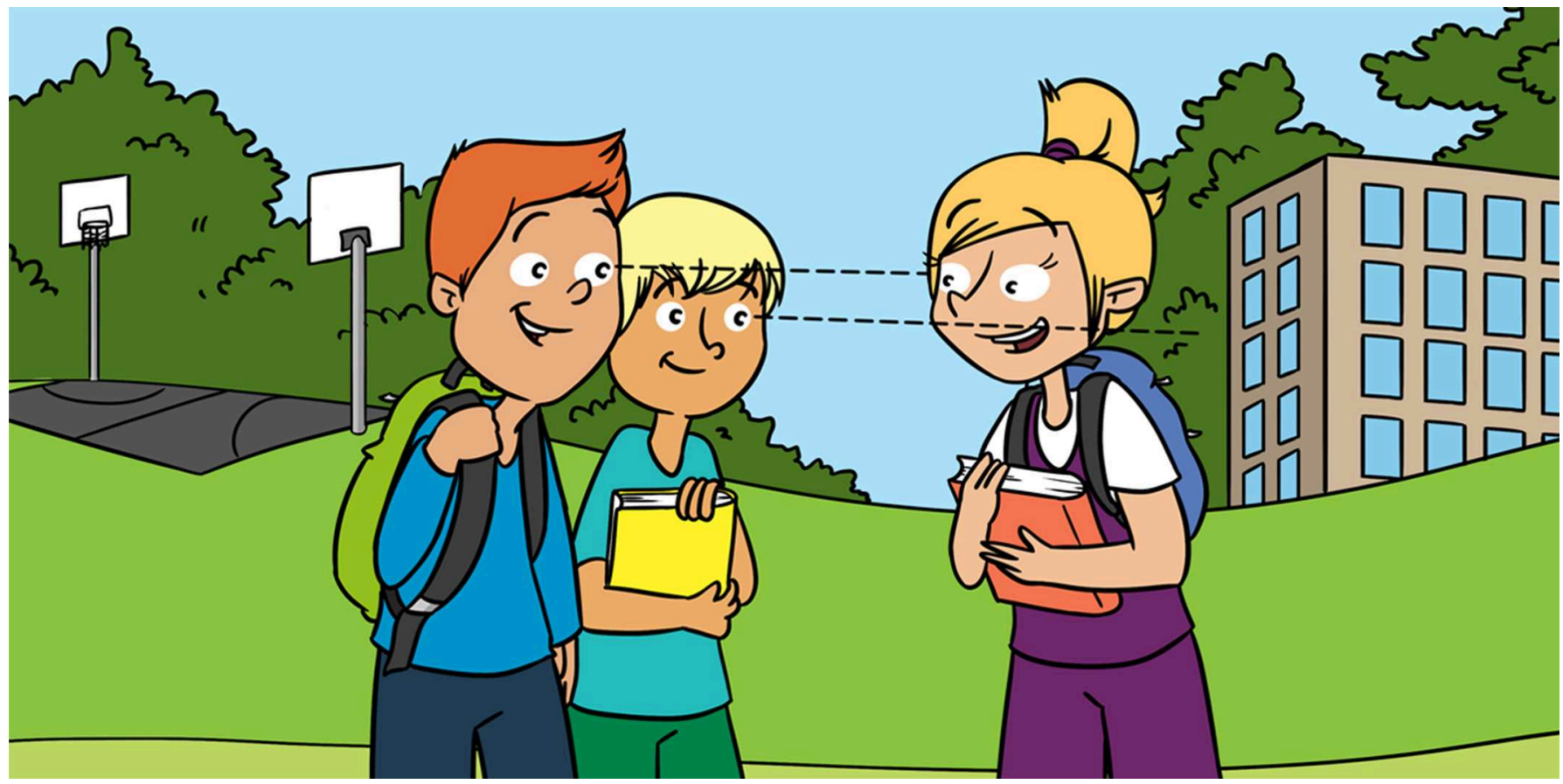

\title{
DOES EVERYONE PAY ATTENTION TO PEOPLE IN THE SAME WAY?
}

\section{Devon S. Heath and Dana A. Hayward *}

Visual Attention and Social Processes Lab, Department of Psychology, University of Alberta, Edmonton, AB, Canada

\section{YOUNG REVIEWERS:}

EXPLORA

SCIENCE

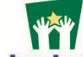

iexploral

Idees You Con Touch
Ideas que puededs tocor
CENTER AND

CHILDREN'S

MUSEUM

AGES: 8-14

\section{ATTENTION}

The process of concentrating on specific information.
The environment around us is constantly changing, so how does the brain adapt to these new and ever-changing situations? Attention helps us filter out distracting information and focus on specific bits of information. For example, when you are at home doing your homework, you might not notice background noises like a dog barking or traffic outside. Not only are researchers interested in how the brain pays attention, but also in how attention is unique for different people and for different types of information, from simple flashes of light to complex faces. In this article, we will explain the importance of social information, how attention prioritizes social information, and how the amount of attention devoted to social information (known as social attention) varies across different people.

\section{WHAT IS SO SPECIAL ABOUT ATTENTION?}

People use some form of attention all the time. By filtering out any unimportant background information, attention helps you focus on 
Figure 1

(A) Newborns were shown a simple face (left) and a scrambled face (right). Researchers found that newborns preferred to look at the simple face, suggesting faces are important early on in our lives! (B) Side view of the left side of the brain. The blue area shows the location of the superior temporal sulcus and the purple area shows the location of the fusiform gyrus. These two areas are active when we look at faces and eyes.
A

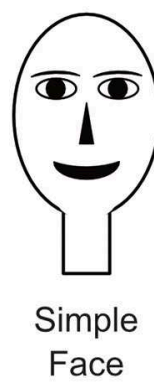

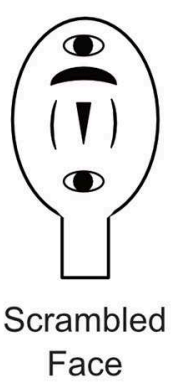

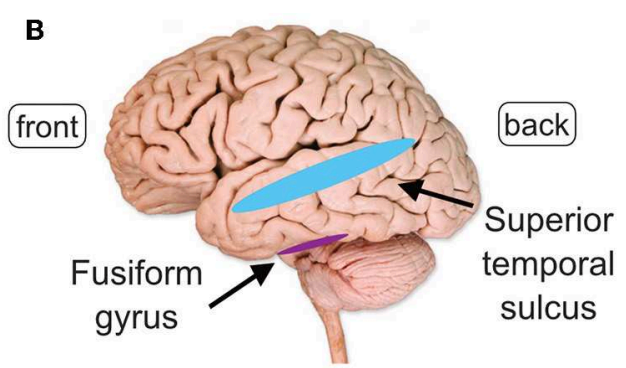

Figure 1

different tasks like drawing a complex scene, playing video games, or reading this article. Attention can also help direct you to especially important information, like a siren or your name, so attention is a very necessary skill for humans. Imagine how difficult it would be if you wanted to focus your attention on something important but could not, or if your attention could not alert you to important changes in the environment. You would not be able to do your homework, learn a new skill, or even cross a busy street!

Because attention is so important, researchers are curious about how the brain knows what to pay attention to, especially when there are so many things happening around us. Some research shows that attention differs from person to person and changes depending on what people are paying attention to. For example, while some individuals pay more attention to social information (this is called social attention), not everyone has the same bias for social attention, which makes researchers interested in the root of these differences.

\section{WHAT IS SO SPECIAL ABOUT SOCIAL INFORMATION LIKE FACES AND EYES?}

Researchers in different fields have found at least two main pieces of evidence to suggest that we see faces, especially eyes, as special. By special, we mean that people usually pay more attention to faces and eyes than to other objects in the world. One piece of evidence comes from studying babies that are only a few hours old. Researchers showed newborns a drawing of a simple face and slowly moved it in front of the babies to see if they followed the face [1]. To make sure babies follow faces and not just moving objects, the researchers also showed the babies a scrambled face. Because the scrambled face has all the same parts as the intact face, researchers can be sure that it is the special layout of a face that is capturing the newborns' attention. Interestingly, babies followed the drawing of the face (Figure 1A, left), but not the scrambled face (Figure 1A, right), showing that faces are important even to babies that are only a few hours old! 
Figure 2

Sequence of the cueing task to measure social attention. Participants first see a small cross in the center of the computer screen. Then a face appears on the screen, looking left or right. Finally, an object (known as a target) appears, either in the location the face is looking (known as a cued trial) or in another location (known as an uncued trial).

Participants respond as quickly as possible when they see the target. Faster responses for cued trials suggest that attention has shifted in the direction that the face is looking [4].

\section{FUNCTIONAL}

MAGNETIC

\section{RESONANCE}

\section{IMAGING (FMRI)}

A brain imaging technique that is used to measure

brain activity.

\section{CUEING TASK}

A computer task that presents an object, like a face, to cue a location in space (such as left or right), followed by a target that can appear in the cued or uncued location. The task measures shifts in attention.

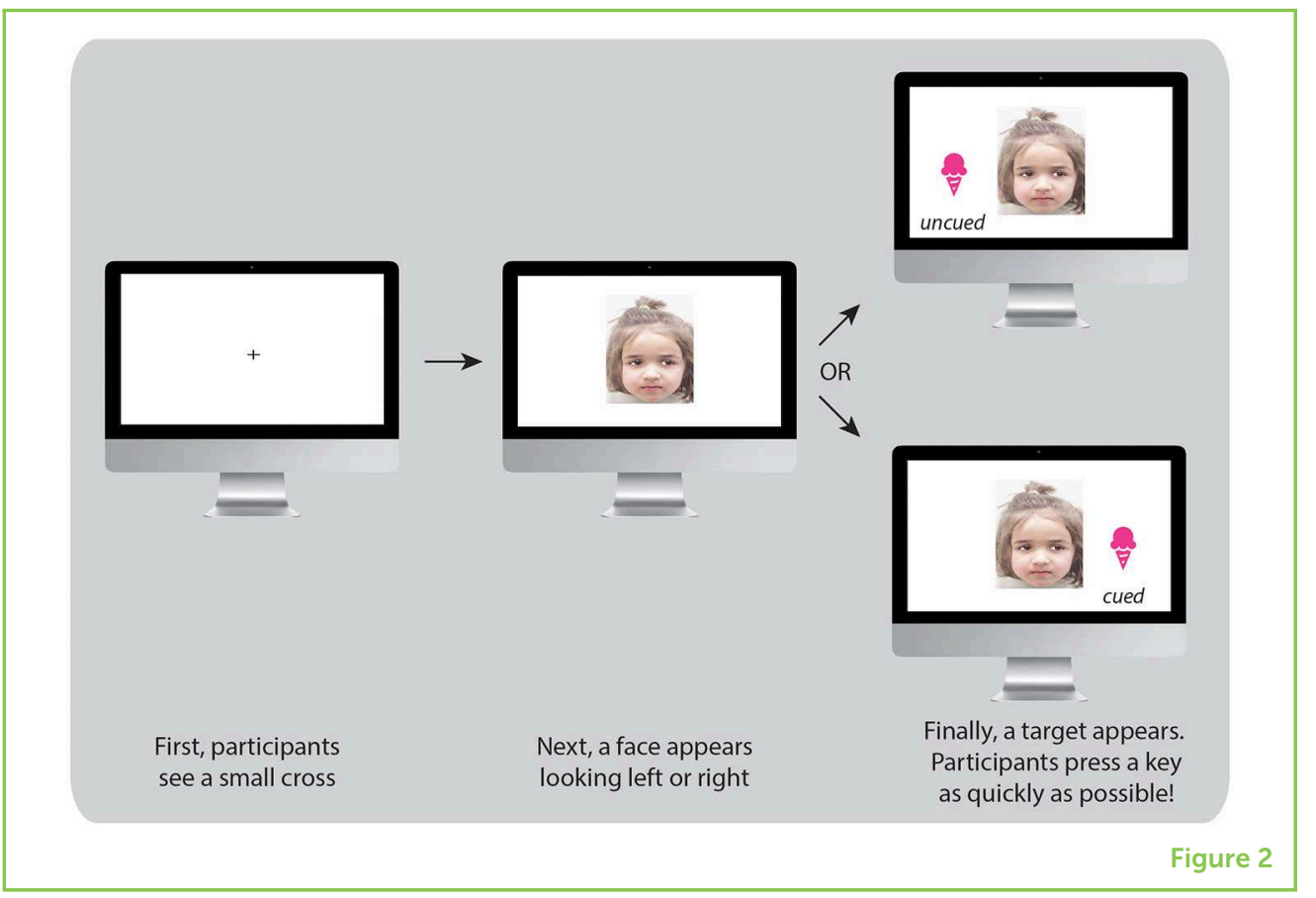

The second piece of evidence that faces and eyes are special comes from studying the brains of people when they look at or pay attention to faces and eyes. Researchers use a technique known as magnetic resonance imaging (MRI) to see both the structure of the brain, and activity of the brain [known as functional magnetic resonance imaging (fMRI)], through measuring where blood flows in the brain during events like looking at faces or paying attention to people. Blood is responsible for bringing more oxygen to the areas of the brain that are very active. This means that we can use fMRI to measure areas of the brain that are active when people are looking at faces! fMRI recordings from adults paying attention to faces and eyes show a lot of activity in parts of the brain called the fusiform gyrus and the superior temporal sulcus (Figure 1B) [2]. This shows that specific parts of our brains are devoted to responding to faces and eyes, again suggesting that these physical features are special.

\section{HOW DO WE MEASURE ATTENTION TO FACES/EYES IN THE LABORATORY?}

One way that researchers measure attention to faces and eyes in the laboratory is by using simple computer tasks [3]. In one of these, called the cueing task, researchers use cues to direct the participant's attention. The sequence of steps (known as a trial) is shown in Figure 2 and in the corresponding movie (Video 1). In this task, first a face appears on the screen, looking either left or right. Next, a target (for example, an ice cream cone) appears either in the location the face is looking at (known as a cued target) or in another location (known as an uncued target). The participant's job is to press a button 


\section{VIDEO 1}

A visual example of the cueing task. In the cueing task, participants keep their eyes fixated in the middle of the screen and respond to targets (i.e., the ice cream) that appear on the left or right side of the face by pressing a button as quickly as possible. Comparing how fast participants respond for cued and uncued trials allows researchers to quantify social attention.

\section{CUED VS. UNCUED}

\section{TARGETS}

In the cueing task, a cue, like a face can either look toward a target (like the ice cream in Figure 2), or away from the target. When the face looks at the target, this is a cued trial, and when the face looks away from the target, this is an uncued trial.

\section{SOCIAL CUEING} EFFECT

A measure of how much faster people respond to targets that are cued by the direction of the face/eyes compared with targets that are uncued. A big difference in speed is considered a large social cueing effect, and a small difference in speed is considered a small social cueing effect.

as soon as they notice the target. Researchers record how long it takes the participant to respond, which is called reaction time. To find out if attention can be shifted by another person's gaze, researchers compared how fast people responded to cued and uncued targets. The results showed that participants responded more quickly to the cued targets (the ones in the direction of the gaze) than to the uncued targets. This means that the participants' attention was shifted by the gaze cue $[5,6]$. This is called the social cueing effect and it shows that people spontaneously pay attention to social things, like faces and eyes. Interestingly, though, research shows that the social cueing effect is smaller for some people and bigger for others.

\section{DOES EVERYONE PAY ATTENTION TO FACES AND EYES IN THE SAME WAY?}

The short answer is no! The same way that people have different interests, strengths, and weaknesses, not everyone pays attention to eyes the same way. For example, people with autism spectrum disorders (ASD) often pay attention to social information in a different way than other groups of people do. Two key features of ASD are difficulties with social situations and the tendency to perform movements or behaviors in very specific or repeated ways [7]. In real life, people with ASD may not look at the eyes of another person as often as someone without ASD does, they may find it difficult to start or respond to conversations, or they may be less interested in social situations [7]. Individuals with ASD are diagnosed by trained doctors who will get reports from daycare providers, teachers, and parents, as well as observe the children and ask them to do various tasks. People with ASD are not completely different from people without ASD. In fact, a group of researchers created a questionnaire called the autism spectrum quotient questionnaire ( $A Q$ ) that allows them to measure the number of autism traits that people have [8]. For example, the questionnaire asks people to rate how true statements about social skills and communication are for them, such as "I enjoy meeting new people," and "I know how to tell if someone listening to me is getting bored." People with ASD are less likely to say these statements are true than are people without ASD.

Researchers have asked how people with different levels of $A Q$ traits, or people with ASD, perform on the social cueing task. Some studies have looked at whether the number of autism traits changes the social cueing effect, and overall it seems that people with more autism traits have a smaller social cueing effect, meaning that they do not prioritize attention to social cues as much as people with fewer autism traits do. For example, in one study, Hayward and Ristic [6] found that smaller social cueing effects were more likely for males, people who have more autism traits according to the $A Q$, and people who have smaller networks of friends. 
Figure 3

(A) A table showing social cueing effects for individuals with and without autism. $(\checkmark)$ Shows the presence of a social cueing effect and $(\chi)$ shows its absence. (B) Data illustrating social cueing effects for individuals with ASD (black bars) and without ASD (gray bars). The useful faces, where targets were looked at most of the time, are shown by the two left bars, while the useless faces, where targets were looked at only some of the time, are depicted by the two right bars. These findings suggest that people without ASD have a strong bias to pay attention to faces, while people with ASD will pay attention to faces only in some situations.

\section{AUTISM SPECTRUM DISORDER}

A group of brain development disorders that affect a person's ability to interact with other people, and cause movements or behaviors to be performed in very specific or repeated ways.

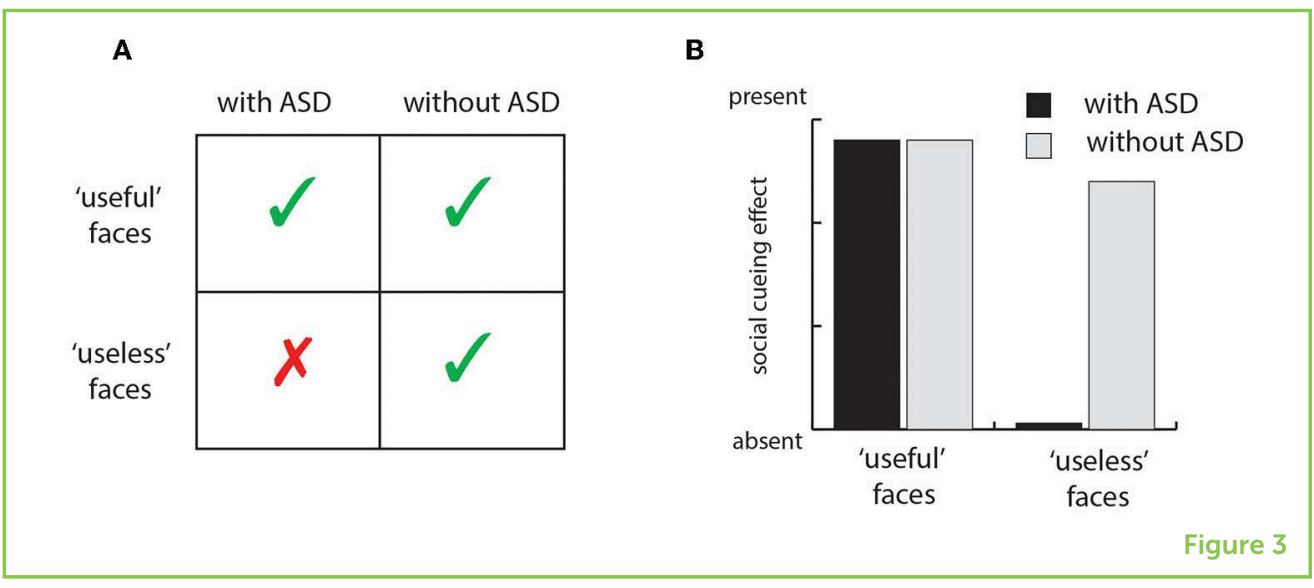

However, when we look at social cueing studies with people with ASD, research is mixed. While many researchers have found differences in attention to faces between people with and without ASD, some researchers have found no differences. One clever study [9] used the social cueing task to investigate how people with and without ASD respond to targets that are almost always looked at by a face, or only looked at some of the time. When the faces almost always looked at the target they were useful to pay attention to. When the faces only looked at the targets sometimes, they were not useful to pay attention to. The researchers found that people without ASD could not help but pay attention to both the "useful" and "useless" faces, showing a bias to pay attention to eyes in both situations (showing a social cueing effect). People with ASD, however, only paid attention to the "useful" faces, and were able to ignore the "useless" faces (Figure 3). These data suggest that attention to faces and eyes is quite strong for people without ASD, but for individuals with ASD, they can choose whether to pay attention to the eyes or not. This means that people with ASD are better able to control their attention, and only pay attention to objects like eyes when they are believed to be useful [9].

\section{CONCLUSION}

People use social attention every day to navigate through their lives. Researchers can measure social attention in the lab and social attention has been found to vary from person to person. Sometimes social attention varies by personality traits, like those measured by the AQ. People with ASD were thought to have different priorities when it comes to social attention, but as we saw from the research [9], this is not always the case. It is not that people with ASD pay attention to social information in a completely different way than people without ASD do, but rather there are differences in how biased we are toward paying attention to social information. These findings help us to see how important paying attention to others can be when we are forming and maintaining social relationships and also help us to better understand why social situations are easier for some people 
than others. Hopefully, knowing that everyone pays attention to social information in different ways will allow us to be more understanding and helpful when we communicate with others.

\section{REFERENCES}

1. Johnson, M. H., Dziurawiec, S., Ellis, H., and Morton, J. 1991. Newborns' preferential tracking of face-like stimuli and its subsequent decline. Cognition 40:1-19. doi: 10.1016/0010-0277(91)90045-6

2. Nummenmaa, L., and Calder, A. J. 2009. Neural mechanisms of social attention. Trends Cogn. Sci. 13:135-43. doi: 10.1016/j.tics.2008.12.006

3. Posner, M. I. 1980. Orienting of attention. Q. J. Exp. Psychol. 32:3-25. doi: 10.1080/00335558008248231

4. LoBue, V., and Thrasher, C. 2015. The child affective facial expression (CAFE) set: validity and reliability from untrained adults. Front. Psychol. 5:1532. doi: 10.3389/fpsyg.2014.01532

5. Friesen, C. K., and Kingstone, A. 1998. The eyes have it! Reflexive orienting is triggered by nonpredictive gaze. Psychon. B Rev. 5:490-5. doi: 10.3758/BF03208827

6. Hayward, D. A., and Ristic, J. 2017. Feature and motion-based gaze cuing is linked with reduced social competence. Sci. Rep. 7:44221. doi: $10.1038 /$ srep44221

7. American Psychiatric Association. 2013. Diagnostic and Statistical Manual of Mental Disorders. 5th ed. Arlington, VA: American Psychiatric Publishing.

8. Baron-Cohen, S., Hoekstra, R. A., Knickmeyer, R., and Wheelwright, S. 2006. The autism-spectrum quotient (AQ)-adolescent version. J. Autism Dev. Disord. 36:343-50. doi: 10.1007/s10803-006-0073-6

9. Ristic, J., Mottron, L., Friesen, C. K., larocci, G., Burack, J. A., and Kingstone, A. 2005. Eyes are special but not for everyone: the case of autism. Cogn. Brain Res. 24:715-8. doi: 10.1016/j.cogbrainres.2005.02.007

SUBMITTED: 20 May 2019; ACCEPTED: 12 November 2019;

PUBLISHED ONLINE: 20 December 2019.

EDITED BY: Kathleen Y. Haaland, University of New Mexico, United States

CITATION: Heath DS and Hayward DA (2019) Does Everyone Pay Attention to People in the Same Way? Front. Young Minds 7:130. doi: 10.3389/frym.2019.00130

CONFLICT OF INTEREST: The authors declare that the research was conducted in the absence of any commercial or financial relationships that could be construed as a potential conflict of interest.

COPYRIGHT @ 2019 Heath and Hayward. This is an open-access article distributed under the terms of the Creative Commons Attribution License (CC BY). The use, distribution or reproduction in other forums is permitted, provided the original author(s) and the copyright owner(s) are credited and that the original publication in this journal is cited, in accordance with accepted academic practice. No use, distribution or reproduction is permitted which does not comply with these terms. 

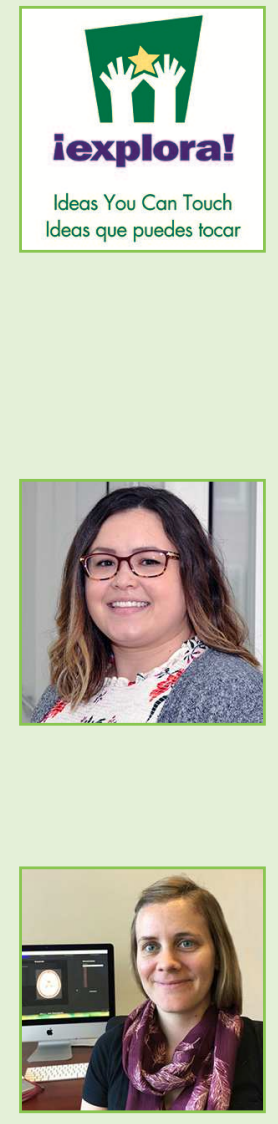

\section{YOUNG REVIEWERS}

\section{EXPLORA SCIENCE CENTER AND CHILDREN'S MUSEUM, AGES: 8-14}

The Explora Young Minds reviewers are a group of science enthusiasts working with museum educators and mentors from the University of New Mexico. We enjoy learning about the brain through the articles. We also enjoy asking questions and making suggestions to help the scientists make their work more understandable for everyone! We were helped by our Science Mentor Stephanie Gorman.

\section{AUTHORS}

\section{DEVON S. HEATH}

I recently received my undergraduate degree in psychology. Now I am working toward becoming a clinician-scientist. I have always been interested in how the brain works and how people differ from one another. I am also passionate about teaching and learning about the brain. In my free time, I like to play video games on my computer! Some of my favorite video games are Fallout, the Elder Scrolls, and League of Legends.

\section{DANA A. HAYWARD}

I am an Assistant Professor in the Psychology Department at the University of Alberta in Canada. In the lab, we study all things related to attention, including how attention changes in real-life situations or in groups, and the effect of rewards on attention. When I am not working, I love to read (to myself and my kids!), play board games, watch movies, and go on fun adventures with my family. *dana.hayward@ualberta.ca 